\title{
Epidemiological trend of pulmonary thromboembolism at a tertiary hospital in Korea
}

\author{
Tae Yun Park ${ }^{\star}$ Jae-Woo Jung ${ }^{\star}$, Jae Chol Choi, Jong Wook Shin, Jae Yeol Kim, Byoung Whui Choi, \\ and In Won Park
}

Department of Internal Medicine, Chung-Ang University Hospital, Seoul, Korea

Received: July 30, 2016 Revised : October 30, 2016 Accepted: November 7, 2016

\section{Correspondence to}

In Won Park, M.D.

Department of Internal Medicine, Chung-Ang University Hospital, 102 Heukseok-ro, Dongjak-gu, Seoul o6973, Korea

Tel: +82-2-6299-1401

Fax: +82-2-6299-2017

E-mail:iwpark@cau.ac.kr

*These authors contributed equally to this work.
Background/Aims: Despite increasing interest in pulmonary thromboembolism (PTE), data on recent trends in PTE incidence are limited. This study evaluated the recent incidence rate of PTE.

Methods: We performed a retrospective chart review of patients with PTE admitted to Chung-Ang University Hospital during the 10-year period from 2006 to 2015. Age-standardized incidence and mortality rates were calculated by the direct method per 100,000 populations. To analyze the trend of risk factor, we also calculated the proportions of cancer, major operation, and recent major fracture over that time.

Results: Total crude incidence rate of PTE per 100,000 was 229.36 and the agesex adjusted standardized incidence rate was 151.28 ( $95 \%$ confidence interval [CI], 127.88 to 177.10). The incidence rate have been significantly increased 1.083 times annually from 2006 (105.96 per 100,000) to 2015 (320.02 per 100,000) (95\% CI, 1.049 to $1.118 ; p<0.001)$. These incidences also increased annually in age group of 35 to 54,55 to 74 , and $\geq 75$ years, and in both males (odds ratio [OR], 1.071; 95\% CI, 1.019 to $1.127 ; p=0.007$ ) and females (OR, 1.091; 95\% CI, 1.047 to 1.136; $p<0.001)$. Cancer accounted for most of the increase from $20.0 \%$ at 2006 to 2007 to $42.8 \%$ at 2014 to 2015 (OR, 1.154; 95\% CI, 1.074 to 1.240; $p<0.001$ ), while the proportions of recent fracture and major operation remained constant.

Conclusions: The incidence of pulmonary embolism has gradually increased over the 10 years. The increase of PTE incidence was mainly due to increased proportion of cancer patients.

Keywords: Pulmonary embolism; Epidemiology; Incidence; Computed tomography; Risk factors

\section{INTRODCTION}

Pulmonary thromboembolism (PTE) is a significant health problem worldwide. In the United States, the prevalence of PTE between 1979 and 1999 was $0.4 \%$ and annual incidence rate was estimated at 600,000 cases [1]. Although the corresponding data for Europe are unavailable, in a community-based study involving 342,000 population in France the incidences of PTE was 6.0 per
10,000 per year [2]. In Australia, the crude annual incidence of PTE is 0.31 per 1,000 residents [3].

Incidence rate of PTE is important because PTE is associated with significant morbidity and mortality in hospitalized patients, as well as economic burden. In the United States, PTE is the third most common cause of death, following heart attack and stroke and the estimated annual economic burden of PTE in the United States exceeds $\$ 8.5$ billion [4-7]. 
Epidemiologic studies of PTE are well established. However, most of the studies were conducted prior to the year 2000 and cannot fully reflect the recent trend of PTE $[2,8,9]$. Another problem is that there is a lack of research on incidence of PTE in Asia compared to Western countries. In Korea, there have only been a few studies addressing the incidence of PTE and little is known about the recent incidence of PTE [10,11]. An updated investigation of PTE is needed.

It is important to understand the recent status of PTE for prevention and treatment of it. Herein, we evaluated the trend of PTE occurrence over a decade in a tertiary hospital in Korea and in national representative data from the Korean National Health Insurance Service (NHIS).

\section{METHODS}

\section{Study population}

PTE patients admitted to Chung-Ang University Hospital from 2006 to 2015 were retrospectively reviewed. All data were gathered in accordance with the amended Declaration of Helsinki, with approval of an independent Institutional Review Board (IRB No: 12-343-394343).

We reviewed records of the patients who were admitted with new diagnosis with PTE and two independent radiologists reviewed chest computed tomography (CT) again on a Radmax PACS work station (Marotech, Seoul, Korea) to reach consensus decisions. For each patient, all electronic medical record data were retrospectively reviewed. Demographic data on the study population including age, sex, body mass index, previous PTE history, combined venous thromboembolism (VTE), comorbidity, history of major operation and recent major fracture, and hospital outcomes were collected. Major operations included orthopedic surgery, major abdomen surgery, major gynecological surgery, major urological surgery, neurosurgery, cardiothoracic, and major vascular surgery. Recent major fracture includes orthopedic injuries, such as long bone fracture and pelvic bone fracture.

\section{Standardization for the Korean population}

For standardization of our hospital population to Korean general population, we used data from the Korean Statistical Information Service (KOSIS, http://kosis.kr/) as the standard population. The Korean population of
KOSIS in 2010 was 48,357,370. The data of age and gender distribution of population in 2010 were used.

\section{Admission rate with PTE and economic burden in Korea}

In order to investigate the annual changes in the total burden of the PTE in Korea, we also analyzed admission rates and following economic burden of admission for PTE using Korean NHIS from 2004 to 2013 . NHIS is the only public medical insurance system operated by the Ministry for Health, Welfare and Family Affairs in Korea, which covers the whole population as a compulsory insurance system [12].

\section{Statistical analyses}

SPSS version 17.0 (SPSS Inc., Chicago, IL, USA) was used for the statistical analyses. Continuous variables are expressed as mean \pm standard deviation (SD) and categorical variables are presented as numbers and percentages. Crude annual incidence rates (per 100,000 individuals) were calculated using the annual number of patients admitted with PTE as the numerator, and the total annual number of admitted patients in Chung-Ang University Hospital for the denominator. To adjust confounders, a multivariate logistic regression analysis involving age and sex was conducted to define the trend in the incidence of PTE to calendar year, and the odds ratio (OR) and $95 \%$ confidence interval (CI) were calculated. Trend in the incidence of PTE according to sex and age group (divided into four groups: o to 34,35 to 54,55 to 74 , and $\geq 75$ years) were also analyzed. Age and sex standardized incidence rates were calculated by the direct method on the KOSIS 2010 standard population. Trend in the incidence of PTE according to calendar year were analyzed by Poisson regression adjusted by age and sex. CI estimates were based on the Poisson distribution. We also calculated the proportion of cancer, major operation, and recent major fracture in patients with PTE to analyze the main cause of PTE trend. Significance was defined as $p<0.05$ for all analyses.

\section{RESULTS}

\section{Baseline characteristics}

Table 1 shows baseline characteristics of study popula- 
Table 1. Baseline characteristics of patients with pulmonary thromboembolism $(n=591)$

\begin{tabular}{|c|c|}
\hline Characteristic & Value \\
\hline Male sex & $224(37.9)$ \\
\hline Age, yr & $68.13 \pm 15.43$ \\
\hline $0-34$ & $27(4.6)$ \\
\hline $35-54$ & $80(13 \cdot 5)$ \\
\hline $55-74$ & $243(41.1)$ \\
\hline$\geq 75$ & $241(40.8)$ \\
\hline Body mass index, $\mathrm{kg} / \mathrm{m}^{2}$ & $28.06 \pm 104.35$ \\
\hline Previous PTE & $8(1.4)$ \\
\hline Combined VTE & $183(31.0)$ \\
\hline \multicolumn{2}{|l|}{ Comorbidities } \\
\hline Hypertension & $239(40.4)$ \\
\hline Cancer & $190(32.1)$ \\
\hline Diabetes & $111(18.8)$ \\
\hline Cerebrovascular disease & $54(9.1)$ \\
\hline Heart failure & $41(7.0)$ \\
\hline Atrial fibrillation & $30(5.1)$ \\
\hline Nephrotic syndrome & $2(0.3)$ \\
\hline Major operation & $151(25 \cdot 5)$ \\
\hline Recent major fracture & $47(8.0)$ \\
\hline \multicolumn{2}{|l|}{ Outcomes } \\
\hline Hospital duration & $30.46 \pm 58.28$ \\
\hline ICU admission & $28(4.7)$ \\
\hline In-hospital death & $46(7.8)$ \\
\hline
\end{tabular}

Values are presented as number (\%) or mean \pm SD.

PTE, pulmonary thromboembolism; VTE, venous thromboembolism; ICU, intensive care unit.

tion. A total of 591 patients were diagnosed with PTE. There were 224 male patients (37.9\%) and the mean age was $68.13 \pm 15.43$ years. The most common comorbidities were hypertension (40.4\%) and cancer (32.1\%). One hundred and fifty-one patients had a recent major operation (25.5\%). Recent fracture accounted for 47 cases (8.0\%). Mean hospital duration day was $30.46 \pm 58.28$ days and intensive care unit admission was 28 cases (4.7\%). Forty-six patients (7.8\%) died in hospital.

\section{Annual incidence rate of PTE}

A total of 257,669 patients were admitted to Chung-Ang University Hospital during the 10-year. Total crude incidence rate of PTE was 229.36 per 100,000. Except for
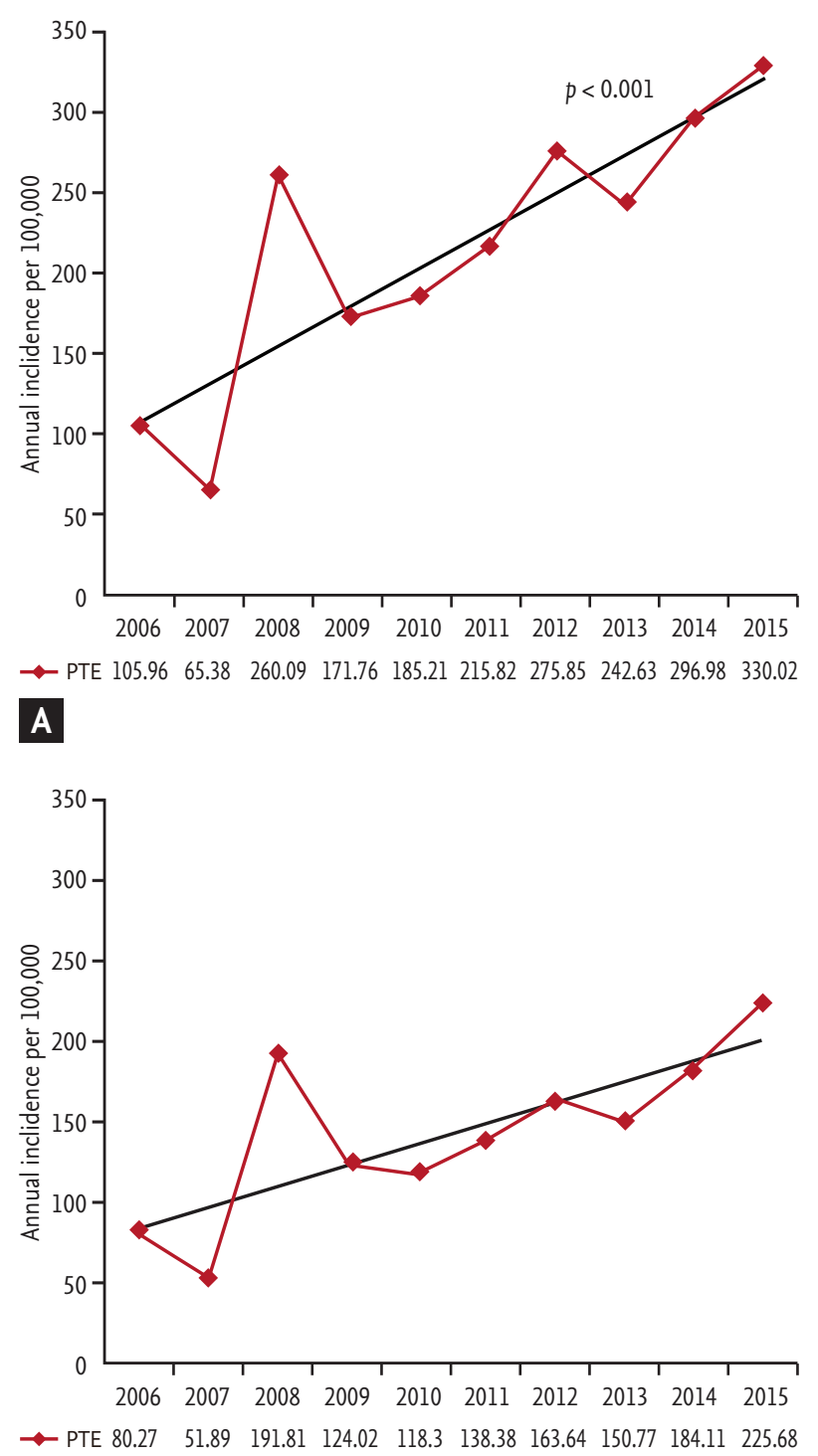

B

Figure 1. The incidence rate of pulmonary thromboembolism (PTE). (A) The crude incidence rate of PTE. (B) The standardized incidence rate of PTE. The data were directly age and sex adjusted to the 2010 standard population of the Korean Statistical Information Service.

an unusual increase of PTE in 2008, incidence rates per 100,000 gradually rose from 105.96 to 320.02 (Fig. 1A). The incidence rate of PTE have been increased 1.083 times annually from 2006 to 2015 , which was statistically significant (95\% CI, 1.049 to 1.118; $p<0.001$ ).

When we standardized crude incidence for age and sex on the KOSIS standard population, the standardized incidence rate of PTE was 151.28 (95\% CI, 127.88 to 177.10). Standardized incidence rates continuously in- 

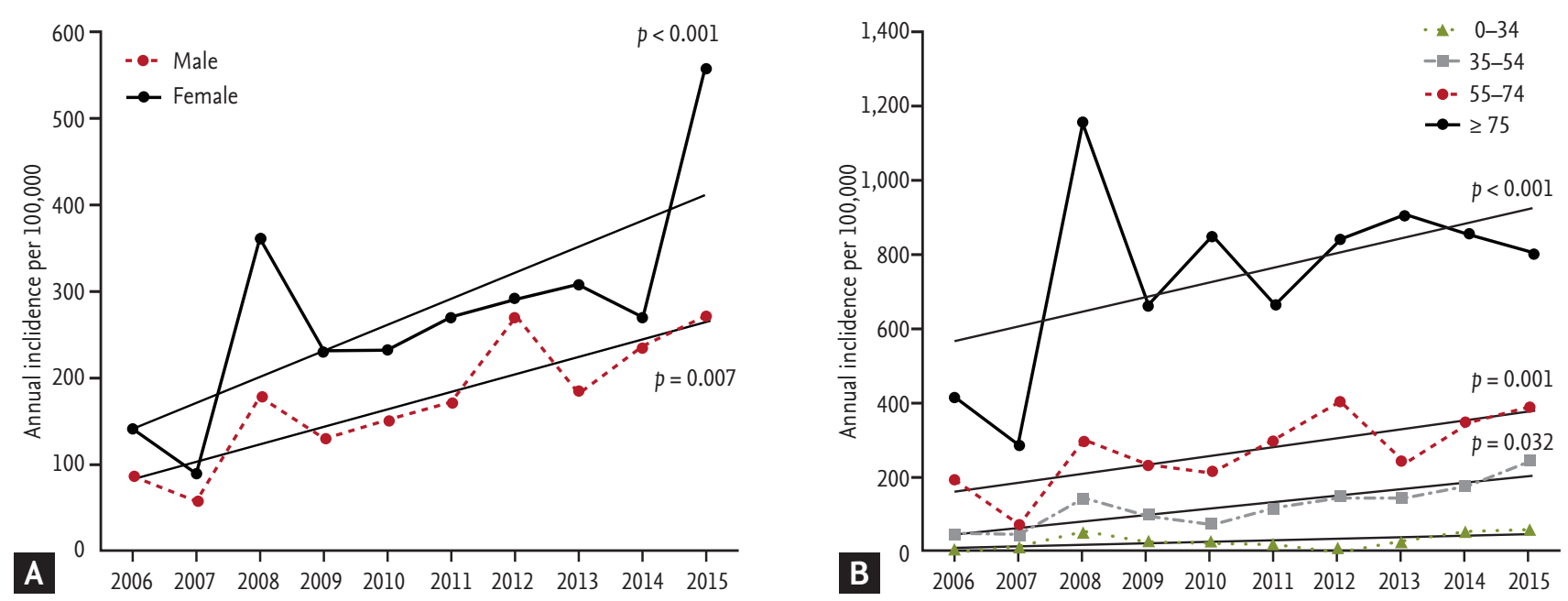

Figure 2. The incidence rate of pulmonary thromboembolism (PTE) according to sex and age group. (A) The incidence rate of PTE according to sex. (B) Annual incidence rate of PTE among different age groups.

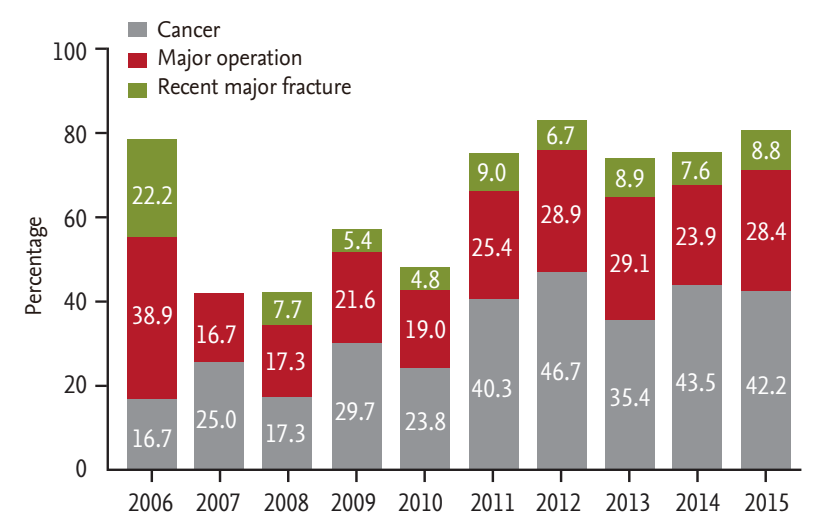

Figure 3. Proportion of cancer, major operation, and recent major fracture in patients with pulmonary thromboembolism.

creased from 80.27 (95\% CI, 63.44 to 99.57 ) to 225.68 (95\% CI, 197.49 to $257 \cdot 47$ ) per 100,000 (Fig. 1B).

\section{Admission rate for PTE and following economic burden in Korea using NHIS data}

In order to confirm the annual changes in the burden of the PTE in Korea, we analyzed the NHIS data. In the NHIS population, the admission rate for PTE per 100,000 during 10 years was 4.92. The annual admission rate per 100,000 was continuously increased from 2.63 in 2004 to 7.44 in 2013 (OR, 1.130; 95\% CI, 1.125 to 1.135; $p<0.001)$. With increased admission rates for PTE, the overall cost for hospitalization was also steadily increased over time (Table 2). In comparison with admis- sion rate of other respiratory disease, the rate of patients with chronic obstructive pulmonary disease and PTE was gradually increased, while that of other respiratory disease tended to decrease over time (Supplementary Fig. 1).

\section{Annual incidence rate of PTE according to sex and age group}

The crude incidence rate of PTE per 100,000 in males was 79.03 in 2006 and 266.84 in 2015. In women, it was 135.30 in 2006 and 533.34 in 2015. Annual incidence rates were tended to increase in both males (OR, 1.071; 95\% CI, 1.019 to $1.127 ; p=0.007)$ and females (OR, 1.091; $95 \%$ CI, 1.047 to 1.136; $p<0.001$ ) (Fig. 2A). The incidence rate in females was higher than that of men over the time period and the relative risk (RR) was 1.546 (95\% CI, 1.308 to $1.828 ; p<0.001$ ).

Except for the age group of o to 34 years, the incidence rate of PTE significantly increased annually from 2006 to 2015 : 35 to 54 years (OR, 1.171; 95\% CI, 1.072 to $1.278, p$ $<0.001) ; 55$ to 74 years (OR, 1.084; 95\% CI, 1.033 to 1.138; $p$ $=0.001)$; and 75 years (OR, 1.057; 95\% CI, 1.005 to 1.112; $p$ $=0.032$ ) (Fig. 2B). Also, the PTE incidence was increased with age. The RRs compared to the o to 34 year group were 4.341 in the 35 to 54 year group (95\% CI, 2.811 to 6.903; $p<0.001$ ), 9.903 in the 55 to 74 year group (95\% CI, 6.656 to $14.734 ; p<0.001$ ) and 26.110 in the $\geq 75$ year group (95\% CI, 17.513 to $38.928 ; p<0.001$ ) (Fig. 2B). 
Table 2. Annual admission rate for pulmonary thromboembolism and following economic burden in Korean National Health Insurance Service population (2004 to 2013)

\begin{tabular}{lcccccccccc}
\hline & 2004 & 2005 & 2006 & 2007 & 2008 & 2009 & 2010 & 2011 & 2012 & 2013 \\
\hline Admission rate $^{\mathrm{a}}$ & 2.64 & 2.76 & 3.09 & 3.91 & 4.61 & 5.02 & 5.87 & 6.58 & 6.97 & 7.44 \\
Overall cost, \$ & $3,101,752$ & $3,590,245$ & $4,114,046$ & $5,714,766$ & $7,097,968$ & $7,986,471$ & $9,411,793$ & $10,255,782$ & $11,501,420$ & $12,523,608$ \\
\hline
\end{tabular}

${ }^{\mathrm{a}}$ All rates are per 100,000 populations.

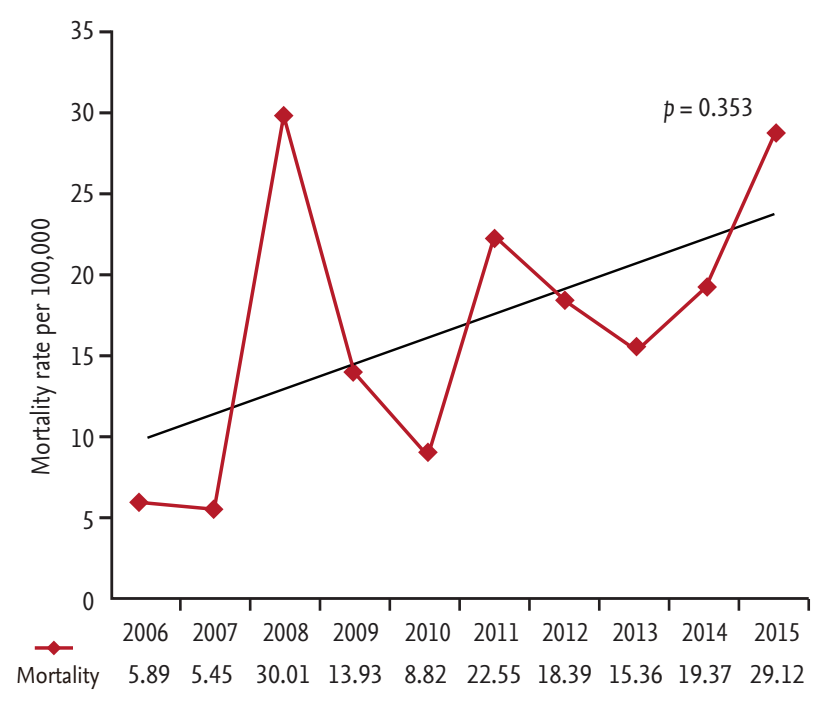

A

Figure 4. In-hospital mortality rate of pulmonary thromboembolism (PTE). (A) The crude in-hospital mortality rate of PTE. (B) The standardized mortality rate of PTE. The data were directly age and sex adjusted to the 2010 standard population of the Korean Statistical Information Service.

\section{Proportion of cancer, major operation, and recent major fracture in patients with PTE}

The proportion of cancer in patients with PTE were gradually increased according to years after adjusted age and sex from $16.7 \%$ at 2006 to $42.2 \%$ at 2015 (OR, 1.154; 95\% CI, 1.074 to 1.240; $p<0.001$ ) (Fig. 3). On the other hand, proportions of major operation (OR, 1.035; 95\% CI, 0.960 to $1.115 ; p=0.375)$ and recent major fracture (OR, $0.986 ; 95 \%$ CI, 0.875 to $1.110 ; p=0.811$ ) revealed no change over the year.

\section{Annual in-hospital mortality of patients with PTE}

Forty-six patients (7.8\%) with PTE died from an underlying medical problem. Cancer $(n=16,35 \%)$ was the most common cause of death, followed by pneumonia $(\mathrm{n}=13$, $28 \%), \operatorname{PTE}(n=7,15 \%)$, myocardial infarction $(n=2,4 \%)$, heart failure ( $n=2,4 \%)$, coagulopathy $(n=2,4 \%)$, liver failure $(n=1,2 \%)$, infection $(n=1,2 \%)$, aspiration $(n=1$,

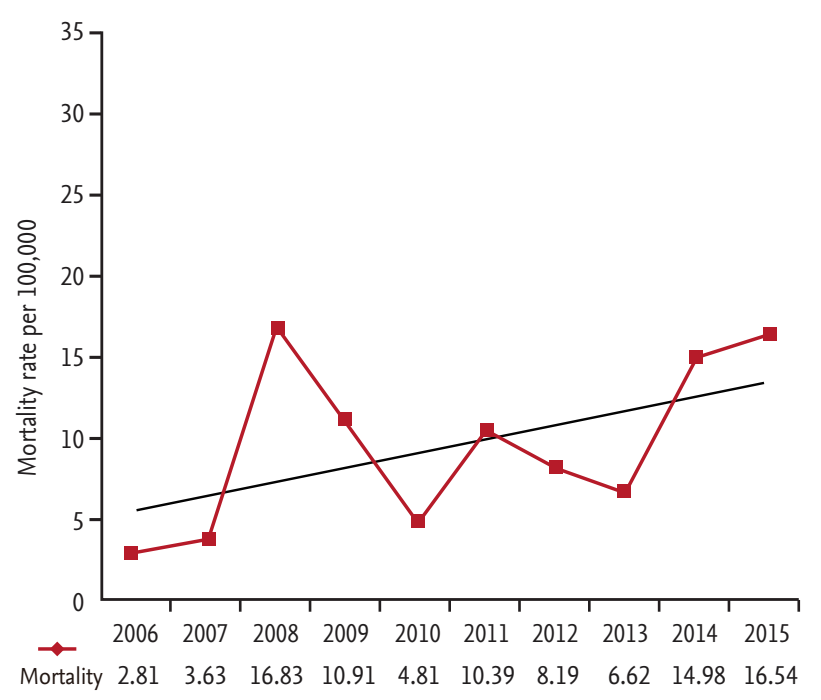

B 
100,000 and the age-sex adjusted standardized incidence rate was 151.28 per 100,000, which was much higher than that of previous studies. Jang at al. [10] reported an incidence of PTE ranging from 3.74 to 7.01 per 100,000 individuals in the Korean Health Insurance Review and Assessment Service (HIRA) database. In the Korean NHIS database, the incidence rate was 4.92 per 100,000, which was more than 47 -fold higher than that of our study. We suggest that the high proportion of high risk patients in acute-care hospitals as the main cause of these results. PTE in a tertiary care hospital is more frequent than general population or short-term hospitals for patients with short admission [13]. Compared with recent tertiary hospital's data, the incidence rate of PTE was 88 (0.17\%) of 50,882 (172.9 per 100,000 patients) in a single center from 2005 to 2007 [11], which was similar to our data.

In addition, the PTE incidence rate continuously increased over the 10-year period in our hospital data as well as NHIS data, like other studies [10,14]. The economic burden also gradually increased over the time, according to NHIS data. Looking at other studies, whereas the incidence rate of PTE tended to decrease before 2000, the incidence rapidly increased since then. Because CT angiography (CTA) is now accepted as the standard method for PTE, the adoption of the technology could have, at least in part, fueled the marked increase in the incidence of PTE in recent years [15]. These studies confirmed that the incidence of PTE was increased according to the use of CTA [15-17].

To evaluate association between increasing incidence and risk factors, we evaluated the trend of risk factors including cancer, major operation, and recent fracture. Cancer is a well-recognized risk factor for VTE and pulmonary embolism [18]. Actually, the cancer incidence was increasing rapidly since 1999 in Korea and this is not only limited in Korea, but is also a global trend [19,20]. To prove this, we calculated the proportions of cancer, major operation, and recent major fracture history in PTE patients. Cancer proportionally increased during the 10-year period. The reason for this result may be also due to the increased use of CT scan in cancer patients. One study confirmed this fact that the widespread use of the recently introduced CT scan lead to increase of incidental PTE in cancer patients [21]. Presently, however, no change was evident in rates of major operation and recent major fractures in our study. The discrep- ancy probably reflects the rapid growth of interest in deep vein thrombosis (DVT) prophylaxis and actual increase of DVT prophylaxis in postoperative and cancer patients [22,23].

In age aspect, the incidence rate of PTE significantly increased annually in all age groups except o- to 34-yearold patients. The PTE incidence was increased depending on age, similar to previous reports $[8,10,11]$. In our study, the patients $\geq 75$ years of age had a 26 times higher PTE risk than patients $<35$ years of age. We suggest that the elderly are exposed to the risk of pulmonary embolism because of both the comorbidities often present with this age group and the accompanying immobility of many elderly people. What is interesting is that age is an important prognostic factor in acute normotensive PTE [24]. Assumes that over-diagnosis is associated with decreasing severity of PTE, old age may be an important factor to predict outcome of PTE. Because the incidence of pulmonary embolism rises with age, we should pay attention to elderly patients.

The effect of sex in PTE has shown conflicting results. Studies of Western populations reported that the incidence of VTE, which is a risk factor of PTE, is higher in men than in women $[3,9]$. However, studies of Korean population have reported that females have a much higher risk of PTE $[10,11]$. Because the issue of a gender effect on is the relationship between female gender and several risk factors, it is difficult to generalize and the effect of gender has not been confirmed so far.

In the same vein as over-diagnosis of PTE, cause-specific mortality was decreased due to decreased severity and mortality was unchanged in a prior study [16]. Although the mortality rate was increased slightly in our study, there was no significant change over time. The most common cause of death was the underlying disease itself, not PTE. Seven patients died from PTE, none since 2014. This was in concordant with the decreasing severity of PTE $[16,17]$.

Our study has some limitations. The study covered hospitalized individuals. Because the population of elderly or patient with severe illness compared to general population, there could have been a selection bias. We adjusted age and sex factors to decrease the effect of population difference and compared it to PTE incidence of the NHIS population.

Despite these limitations, our study provides knowl- 
edge that is valuable to understand the recent trend of PTE. We confirmed this trend of PTE incidence in a tertiary hospital. The increase of PTE incidence was mainly due to the increased proportion of cancer patients. Prospective, multi-center studies are necessary.

\section{KEY MESSAGE}

1. The incidence of pulmonary embolism had gradually increased over the 10 years.

2. Old age and female sex showed increased risk for pulmonary thromboembolism.

3. The increased proportion of cancer patients was associated with the increase of pulmonary thromboembolism.

\section{Conflict of interest}

No potential conflict of interest relevant to this article was reported.

\section{REFERENCES}

1. Stein PD, Beemath A, Olson RE. Trends in the incidence of pulmonary embolism and deep venous thrombosis in hospitalized patients. Am J Cardiol 2005;95:1525-1526.

2. Oger E. Incidence of venous thromboembolism: a community-based study in Western France. EPI-GETBP Study Group. Groupe d'Etude de la Thrombose de Bretagne Occidentale. Thromb Haemost 2000;83:657-66o.

3. Ho WK, Hankey GJ, Eikelboom JW. The incidence of venous thromboembolism: a prospective, community-based study in Perth, Western Australia. Med J Aust 2008;189:144-147.

4. Mahan CE, Borrego ME, Woersching AL, et al. Venous thromboembolism: annualized United States models for total, hospital-acquired and preventable costs utilizing long-term attack rates. Thromb Haemost 2012;108:291302.

5. Goldhaber SZ, Bounameaux H. Pulmonary embolism and deep vein thrombosis. Lancet 2012;379:1835-1846.

6. Agnelli G, Becattini C. Acute pulmonary embolism. N Engl J Med 2010;363:266-274.

7. Kroger K, Kupper-Nybelen J, Moerchel C, Moysidis T, Kienitz C, Schubert I. Prevalence and economic burden of pulmonary embolism in Germany. Vasc Med 2012;17:303309.
8. Anderson FA Jr, Wheeler HB, Goldberg RJ, et al. A population-based perspective of the hospital incidence and case-fatality rates of deep vein thrombosis and pulmonary embolism: the Worcester DVT Study. Arch Intern Med 1991;151:933-938.

9. Silverstein MD, Heit JA, Mohr DN, Petterson TM, O'Fallon WM, Melton LJ 3 rd. Trends in the incidence of deep vein thrombosis and pulmonary embolism: a 25-year population-based study. Arch Intern Med 1998;158:585-593.

10. Jang MJ, Bang SM, Oh D. Incidence of venous thromboembolism in Korea: from the Health Insurance Review and Assessment Service database. J Thromb Haemost 2011;9:85-91.

11. Choi WI, Lee MY, Oh D, Rho BH, Hales CA. Estimated incidence of acute pulmonary embolism in a Korean hospital. Clin Appl Thromb Hemost 2011;17:297-301.

12. Kim DS. Introduction: health of the health care system in Korea. Soc Work Public Health 2010;25:127-141.

13. Stein PD, Huang Hl, Afzal A, Noor HA. Incidence of acute pulmonary embolism in a general hospital: relation to age, sex, and race. Chest 1999;116:909-913.

14. Sakuma M, Takahashi T, Demachi J, et al. Epidemiology of pulmonary embolism in Japan. In: Shirato K, ed. Venous Thromboembolism. Tokyo: Springer, 2005:3-12.

15. DeMonaco NA, Dang Q, Kapoor WN, Ragni MV. Pulmonary embolism incidence is increasing with use of spiral computed tomography. Am J Med 2008;121:611-617.

16. Wiener RS, Schwartz LM, Woloshin S. Time trends in pulmonary embolism in the United States: evidence of overdiagnosis. Arch Intern Med 2011;171:831-837.

17. Schissler AJ, Rozenshtein A, Schluger NW, Einstein AJ. National trends in emergency room diagnosis of pulmonary embolism, 2001-2010: a cross-sectional study. Respir Res 2015;16:44.

18. Shen VS, Pollak EW. Fatal pulmonary embolism in cancer patients: is heparin prophylaxis justified? South Med J 1980;73:841-843.

19. Jemal A, Center MM, DeSantis C, Ward EM. Global patterns of cancer incidence and mortality rates and trends. Cancer Epidemiol Biomarkers Prev 2010;19:1893-1907.

2o. Jung KW, Won YJ, Kong HJ, Oh CM, Seo HG, Lee JS. Cancer statistics in Korea: incidence, mortality, survival and prevalence in 2010. Cancer Res Treat 2013;45:1-14.

21. Abdel-Razeq HN, Mansour AH, Ismael YM. Incidental pulmonary embolism in cancer patients: clinical characteristics and outcome: a comprehensive cancer center 
experience. Vasc Health Risk Manag 2011;7:153-158.

22. Lyman GH, Khorana AA, Falanga A, et al. American Society of Clinical Oncology guideline: recommendations for venous thromboembolism prophylaxis and treatment in patients with cancer. J Clin Oncol 2007;25:5490-5505.

23. Hardwick ME, Colwell CW Jr. Advances in DVT prophylaxis and management in major orthopaedic surgery. Surg Technol Int 2004;12:265-268.
24. Keller K, Beule J, Coldewey M, Geyer M, Balzer JO, Dippold W. The risk factor age in normotensive patients with pulmonary embolism: effectiveness of age in predicting submassive pulmonary embolism, cardiac injury, right ventricular dysfunction and elevated systolic pulmonary artery pressure in normotensive pulmonary embolism patients. Exp Gerontol 2015;69:116-121. 


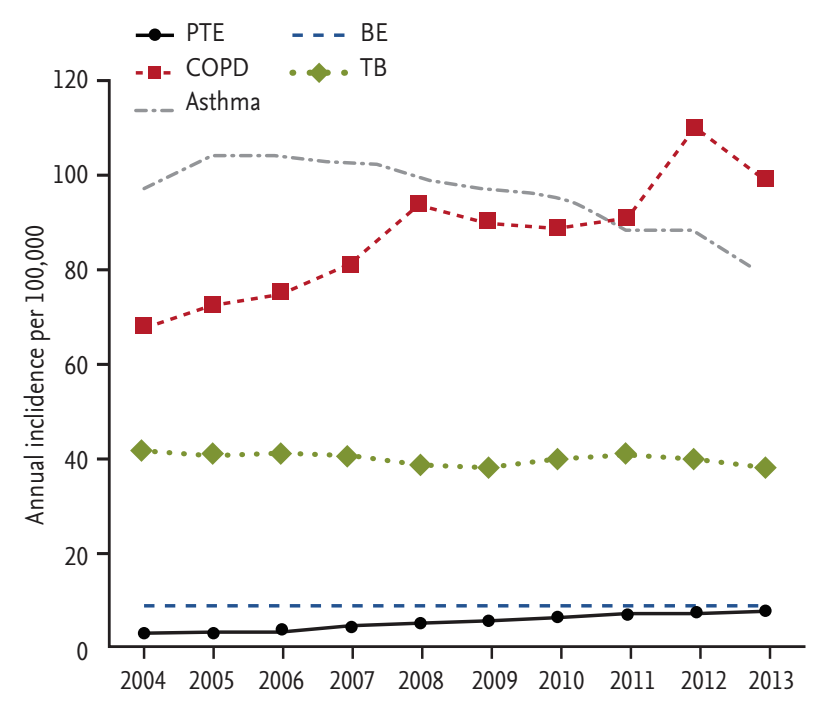

Supplementary Figure 1. Annual admission rate for pulmonary thromboembolism (PTE) and other respiratory disease. COPD, chronic obstructive pulmonary disease; BE, bronchiectasis; TB, tuberculosis. 